13

\title{
Низкотемпературная растворимость гелия в кварце
}

\author{
(C) А.В. Коромыслов, А.Я. Купряжкин \\ Уральский фредеральный университет, \\ 620002 Екатеринбург, Россия \\ e-mail: a.v.koromyslov@gmail.com
}

(Поступило в Редакцию 10 мая 2016 г. В окончательной редакции 8 сентября 2016 г.)

Методом термодесорбции гелия из предварительно насыщенных в газовой фазе кристаллов в диапазоне температур $T=323-1123 \mathrm{~K}$ и давлений насыщения $P=0.6-31 \mathrm{MPa} \mathrm{исследована} \mathrm{растворимость} \mathrm{гелия}$ в монокристаллах $\alpha, \beta$-кварца, стеклах оптического кварца и стекле пирекс. Показано, что поведение растворимости гелия в стеклах кварца описывается экспоненциальными зависимостями от обратной температуры. Растворимость гелия в монокристаллах кварца практически не зависит от температуры. Растворимость гелия для всех типов образцов изменяется пропорционально давлению насыщения и достигает значений $(6.2 \pm 0.3) \cdot 10^{15} \mathrm{~cm}^{-3}$ в монокристаллах при $P=25.5 \mathrm{MPa}, T=873 \mathrm{~K}$ и $(7.01 \pm 0.04) \cdot 10^{19} \mathrm{~cm}^{-3}$ в оптическом кварце при $P=31 \mathrm{MPa} \mathrm{и} T=373 \mathrm{~K}$. Показана возможность роста растворимости гелия в монокристаллах кварца за счет увеличения дефектов кристалла при многократном термоциклировании образцов в процессе измерений. Рассмотрены возможные механизмы растворения гелия в кварце, приведено сравнение с данными других авторов.

DOI: 10.21883/JTF.2017.09.44919.1874

\section{Введение}

Интерес к исследованию растворимости гелия в кварце наряду с его диффузией обусловлен совершенствованием методов геохронологии [1], развитием методов гелиевой дефектоскопии кристаллов [2], разработкой методов получения высококонцентрированных растворов изотопов гелия.

Сложность проведения исследования в кварце обусловлена наличием большого числа его фаз в широкой области температур и давлений [3], присутствием воды на поверхности и границах зерен и дислокаций [4], малым значением растворимости гелия в монокристаллах кварца [5]. Основные исследования растворимости, как правило, проведены в стеклообразном кварце методом проницаемости [6], в том числе для малого диапазона температур [7], и методом термодесорбции для порошкообразных высокодефектных фаз кварца [3], обладающих высокой растворимостью гелия. При этом в работе [6] при температуре $550 \mathrm{~K}$ зарегистрировано изменение энергии растворения, а в работе [7] показана возможность получения разных значений растворимости гелия в кварце в методах проницаемости и термодесорбции из-за различного вклада дефектов объема и поверхности исследуемых образцов кварца на измеряемые величины. При этом полученные в указанной работе энергии растворения гелия имеют относительно высокие значения.

Наиболее последовательные исследования по взаимодействию гелия с дефектами в области существования $\alpha$-фазы в монокристаллах кварца выполнены в работе [5], однако зависимости растворимости гелия от температуры и давления насыщения авторы не изучали. Представляет интерес проведение сравнительных исследований низкотемпературной растворимости гелия в кварцевом стекле и монокристаллах $\alpha, \beta$-кварца, выяснение возможностей гелиевой дефектоскопии для исследования данных образцов.

\section{Методика проведения эксперимента}

В экспериментах использовали образцы стекол оптического кварца и пирекса и образцы монокристалла синтетического кварца, вырезанные из различных участков монокристалла пьезокварца. Характерный размер образцов, используемых в эксперименте, не превышал $2 \mathrm{~mm}$.

Химический анализ исходных образцов (wt.\%) проводили с помощью масс-спектрометра с индуктивно связанной плазмой (ISP MS). Содержание основных примесей $(\mathrm{Fe})$ в образце оптического кварца и монокристаллах не превышало $0.009 \%$, остальных примесей $<0.001 \%$. Содержание основного материала $\mathrm{SiO}_{2}$ в образце пирекса - 80.5\%, основные примеси: В $4.823 \%, \mathrm{Na}-6.348 \%, \mathrm{~K}-0.044 \%, \mathrm{Ca}-0.104 \%, \mathrm{Ti}-$ $0.014 \%, \mathrm{Fe}-0.024 \%, \mathrm{Zr}-0.035 \%$. Остальные примеси составляли $<0.001 \%$.

Исследование растворимости гелия в кварце проводили методом термодесорбции газа из предварительно насыщенных в камере насыщения при заданных температуре и давлении гелия образцов, закаленных после насыщения до комнатной температуры и перегруженных в измерительную камеру для дегазации при высокой температуре $(1200 \mathrm{~K})$. Использование для дегазации монокристаллов температуры меньшей, чем температура плавления кварца, позволило, с одной стороны, исключить из получаемой растворимости вклад от возможных глубоких „ловушек“, связывающих гелий до температуры плавления [5], с другой - дало возможность использовать исходные кристаллы неоднократно, в более широком интервале температур и давлений. 
Измерения количества выделенного гелия проводили с помощью модернизированного масс-спектрометра МИ-1201, работающего в квазистатическом (по гелию) режиме откачки с геттерным насосом. Для калибровки масс-спектрометра использовали метод двойного расширения известного количества гелия из калиброванного объема в предварительно измеренный объем измерительной камеры масс-спектрометра с регистрацией давления в калиброванном объеме аналогично [2]. Время насыщения исследуемых образцов подбирали экспериментально после проверки воспроизводимости результатов при временах насыщения, отличающихся не менее чем в 3 раза.

\section{Результаты экспериментов и их обсуждение}

Результаты измерений температурной зависимости растворимости гелия в оптическом кварце (рис. 1, кривые 1) и стекле пирекс (рис. 1, кривые 2) приведены для исследованного диапазона температур $T=323-1123 \mathrm{~K}$ и давления насыщения $P=1 \cdot 10^{5} \mathrm{~Pa}$. Для сравнения также представлены результаты работ [6,8], выполненных при определении растворимости гелия из исследований проницаемости оптического кварца для исследуемого диапазона температур (рис. 1, кривые 3,4). На рис. 2 представлены результаты измерений растворимости гелия от давлений насыщения для оптического кварца и одного из образцов монокристалла, полученных в настоящей работе. Как следует из сравнения, в отличие от работы [6] поведение растворимости гелия в зависимости от температуры насыщения $\ln C=f(1 / T)$ для каждого из образцов стекол хорошо описывается одной экспонентой (рис. 1, кривые 1,2). Зависимости растворимости гелия от давления насыщения в стекле оптического кварца и монокристалле кварца (рис. 2,

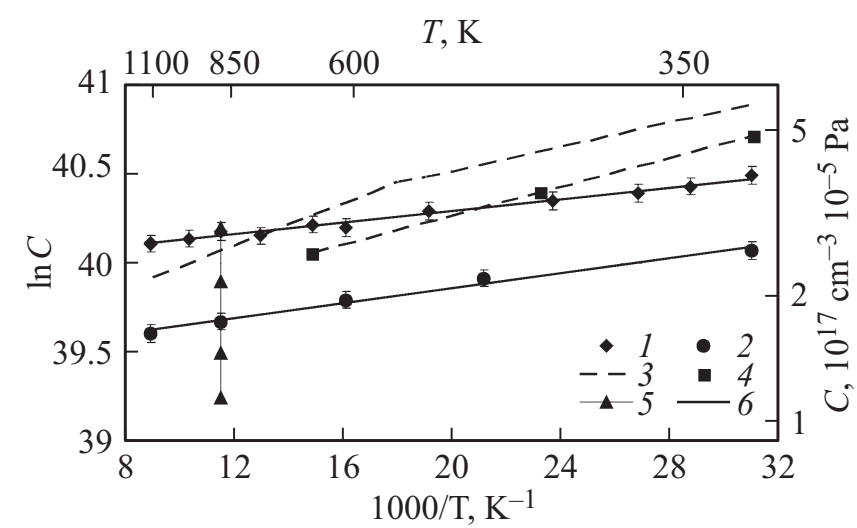

Рис. 1. Растворимость гелия в стеклах кварца, $P=1 \cdot 10^{5} \mathrm{~Pa}$ 1 - в оптический кварц, 2 - пирекс, 3 - данные работы [6], 4 - данные работы [8], 5 - данные по растворимости гелия в оптическом кварце $(T=873 \mathrm{~K})$ после четырех последовательных циклов насыщения-дегазации (см. текст), 6 аппроксимации данных настоящей работы.

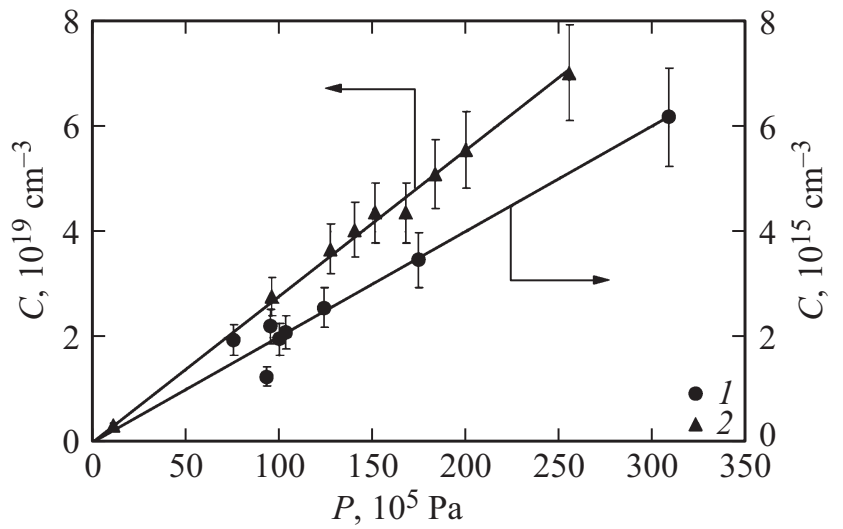

Рис. 2. Зависимость растворимости гелия в кварце от давления насыщения: $1-$ монокристалл кварца $T=873 \mathrm{~K}, 2-$ оптический кварц $T=373 \mathrm{~K}$.

кривые 1,2 ) подчиняются закону Генри:

$$
C=\Gamma(T) P
$$

где $\Gamma(T)$ - константа.

Из рассмотрения условий равновесия в системе газнесовершенный кристалл для зависимости растворимости атомов гелия, находящихся в определенных энергетических позициях кристалла, при заданных температуpe $T$ и малом давлении насыщения $P$, нетрудно получить (см., например, [9]):

$$
C=C^{*} L P \exp (-\Delta E / k T)
$$

где $C^{*}, \Delta E-$ концентрация позиций и энергий растворения гелия в данной позиции, $L-$ константа, слабо зависящая от температуры, $C^{*} L P=C_{0}-$ предэкспоненциальный множитель (2).

Результаты обработки зависимостей (2) для настоящей работы в сравнении с данными других работ $[6,8]$ приведены в таблице. Константы $\Gamma(T)$ для соотношения (1) в соответствии с экспериментом (рис. 2) составили: для оптического кварца $-\Gamma(373 \mathrm{~K})=(2.7 \pm$ $\pm 0.1) \cdot 10^{17} \mathrm{~cm}^{-3} 10^{-5} \mathrm{~Pa}^{-1}$, для монокристалла кварца $\Gamma(873 \mathrm{~K})=(2.0 \pm 0.2) \cdot 10^{13} \mathrm{~cm}^{-3} 10^{-5} \mathrm{~Pa}^{-1}$.

Температурные зависимости растворимости гелия в стеклах оптического кварца (рис. 1, кривая 1) и пирексе (рис. 1, кривая 2) в полулогарифмических координатах практически параллельны и имеют равные в пределах погрешности измерений энергии растворения. Можно предположить, что исследуемые стекла обладают идентичной структурой. Поэтому число свободных позиций для растворения гелия $-C_{i}^{*}$ в пирексе по данным растворимости (2) на 37\% меньше, чем в оптическом кварце из-за наличия в нем различных примесей, занявших изначально свободные позиции в процессе изготовления пирекса. Отрицательные значения энергии растворения в стеклах (таблица, строки 1,2) указывают на то, что характерный размер регистрируемых позиций растворения 
Параметры температурной зависимости растворимости гелия в кварце

\begin{tabular}{|c|c|c|c|c|}
\hline № & Обозначения & $T, \mathrm{~K}$ & $C_{i 0}, \mathrm{~cm}^{-3} 10^{-5} \mathrm{~Pa}^{-1}$ & $\Delta E_{i}, \mathrm{eV}$ \\
\hline \multicolumn{5}{|c|}{ Стекло (обозначения рис. 1) } \\
\hline $\begin{array}{r}1 \\
2 \\
3 \\
4^{*} \\
\end{array}$ & $\begin{array}{c}1 \\
2 \\
3[6] \\
4[6] \\
5[9] \\
\end{array}$ & $\begin{array}{l}323-1123 \\
323-1123 \\
323-550 \\
550-1123 \\
323-673 \\
\end{array}$ & $\begin{array}{c}(2.24 \pm 0.04) \cdot 10^{17} \\
(1.34 \pm 0.04) \cdot 10^{17} \\
1.99 \cdot 10^{17} \\
1.28 \cdot 10^{17} \\
(1.36 \pm 0.01) \cdot 10^{17}\end{array}$ & $\begin{array}{c}-0.015 \pm 0.001 \\
-0.018 \pm 0.001 \\
-0.03 \\
-0.05 \\
-0.0351 \pm 0.0004\end{array}$ \\
\hline \multicolumn{5}{|c|}{ Монокристалл (обозначения рис. 3) } \\
\hline $\begin{array}{l}5 \\
6 \\
7 \\
8\end{array}$ & $\begin{array}{c}1,2 \\
3 \\
4 \\
5\end{array}$ & $\begin{array}{l}573-1123 \\
573-1023 \\
523-1123 \\
573-1123\end{array}$ & $\begin{array}{c}(2.2 \pm 0.3) \cdot 10^{13} \\
(7 \pm 2) \cdot 10^{13} \\
(10 \pm 2) \cdot 10^{13} \\
(1.7 \pm 0.1) \cdot 10^{14}\end{array}$ & $\begin{aligned} 0.009 & \pm 0.008 \\
0.04 & \pm 0.02 \\
0.03 & \pm 0.01 \\
0.006 & \pm 0.005\end{aligned}$ \\
\hline
\end{tabular}

Примеч ание ${ }^{*}$ - обработано нами.

сравним или превышает размер атома гелия $-0.25 \mathrm{~nm}$ (см., например, [9]). Последний вывод подтверждается также данными по удалению летучих примесей, имеющихся изначально в оптическом кварце, в процессе циклов насыщения - дегазация. Так, для исходных (необезгаженных) образцов оптического кварца $(T=873 \mathrm{~K}$, рис. 1, кривая 5) приведены результаты четырех последовательных операций (насыщение, дегазация) по определению растворимости гелия. Первый результат дал самую низкую растворимость (ниже, чем в пирексе). Последующие измерения привели к росту растворимости (рис. 1, кривая 5, указано вертикальной стрелкой) до максимального воспроизводимого значения, что может быть связано с очисткой исследованного образца от летучих примесей и увеличением числа позиций $C^{*}$ соответственно для растворимости гелия (2). Сравнение данных настоящей работы (рис. 1 , кривые 1,2 ) с данными работы [6] (рис. 1, кривые 3,4) указывает, как и в случае $[5,8]$ (рис. 1 , кривая 4), на отсутствие „излома“ на температурной зависимости $\ln C=f(1 / T)$. Наличие последнего может быть обусловлено присутствием в образце неконтролируемых примесей, в частности воды, или особенностями определения растворимости газов из данных метода проницаемости. Повышенное значение энергии растворения, полученное в работе [7], повидимому, обусловлено теми же причинами и, кроме того, малым диапазоном температур измерения.

Для исследования растворимости гелия в монокристаллах вырезали три образца из исходного монокристалла кварца (№ 1-3). Экспериментальные данные по растворимости гелия в них, полученные в области существования $\alpha, \beta$-фаз кварца $573-1123 \mathrm{~K}$, приведены на рис. 3 , кривые $1-3$. Граница раздела фаз обозначена вертикальными штрихами. Из данных рисунка следует, что величины растворимости гелия для исходных образцов № 1,2 совпадают, а для образца № 3 превышают их более чем в 2 раза. Последнее может быть обусловлено растворением гелия в дефектах монокристалла и их неоднородным распределением в исходном образце. Для проверки этого предположения проводили повторное (после первой серии измерений и связанного с ними термоциклирования) измерение растворимости в образце № 3. Полученная растворимость (рис. 3, кривая 4) во всем диапазоне температур возросла в среднем в 1.4 раза по сравнению с исходным образцом № 3. После второго термоциклирования образца (№ 3) с соответствующими измерениями растворимости в образце удалось добиться стабильного увеличения растворимости (рис. 3, кривая 5) по сравнению с исходным в 3.5 раза. Полученные результаты свидетельствуют о том, что рост растворимости гелия в монокристалле кварца обусловлен появлением дополнительных дефектов, наиболее вероятно дислокаций, образующихся в результате накопления упругих напряжений в кристалле при термоциклировании. Этим же можно объяснить стабилизацию роста растворимости после второй обработки кристалла (рис. 3, кривая 5), связанную с исчерпанием возмож-

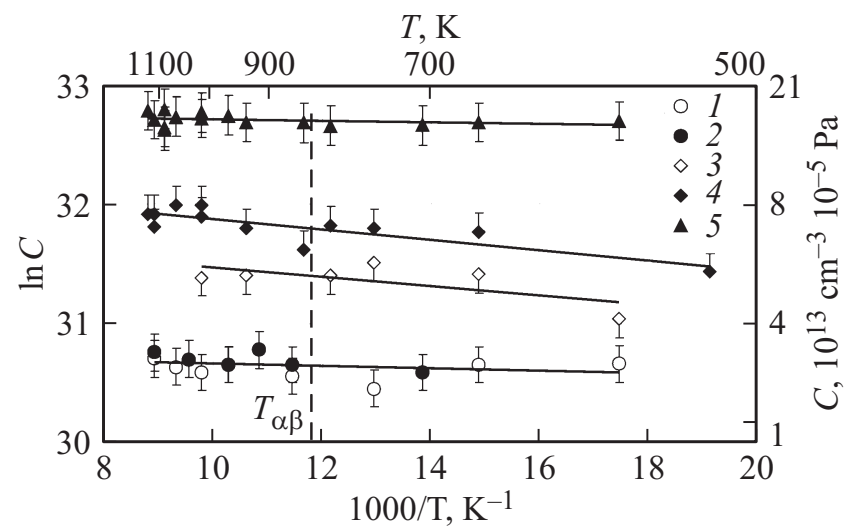

Рис. 3. Растворимость гелия в монокристаллах кварца, $P=1 \cdot 10^{5} \mathrm{~Pa}: 1-3$-данные растворимости гелия в исходных образцах монокристалла кварца № 1, № 2, № 3; 4 растворимость гелия в образце № 3 после однократного термоциклирования; 5 - растворимость гелия в образце № 3 после двукратного термоциклирования. 
ностей термоциклирования для дальнейшего увеличения числа дислокаций.

Из приведенных данных следует также, что представленные зависимости растворимости гелия для всех исследованных образцов монокристаллов кварца (рис. 3) в пределах погрешности измерений имеют пренебрежимо малые отличия при температуре фазового перехода (рис. $3 ; T_{\alpha, \beta}$ (штрихи)) и могут быть аппроксимированы для каждого из образцов во всем интервале исследованных температур 573-1123 К одной аррениусовской зависимостью. Данные обработки температурных зависимостей растворимости гелия в монокристаллах приведены в таблице. Из данных рис. 3 и таблицы следует, что энергии растворения гелия в монокристаллах кварца очень малы, нулевые или положительные, в сравнении с энергиями растворения в стеклах.

\section{Заключение}

Экспериментальные результаты по увеличению растворимости гелия в монокристаллах $\alpha, \beta$-кварца, полученные в настоящей работе после термоциклирования исходных монокристаллов, так же как и малые значения энергий растворения гелия, полученных из температурных зависимостей растворимостей гелия в $\alpha, \beta$-кварце, указывают на возможность дислокационного механизма растворения гелия в монокристаллах $\alpha, \beta$-кварца.

Данные для энергий растворения гелия в $\alpha, \beta$-кварце (таблица) указывают на то, что размер позиций растворения гелия в монокристаллах кварца меньше или равен размеру атома гелия, что также не противоречит дислокационному механизму растворимости, как и тот факт, что минимальное значение растворимости гелия, зарегистрированное в настоящей работе, на порядок выше, чем в работе [5], где эксперименты проведены на монокристаллах с плотностью дислокаций $\left(\sim 10^{2} \mathrm{~cm}^{-2}\right)$.

Наибольшее значение растворимости гелия, полученное в настоящей работе для монокристаллов кварца, $(6.2 \pm 0.3) \cdot 10^{15} \mathrm{~cm}^{-3}$ при $P=31 \mathrm{MPa}, T=873 \mathrm{~K}$. В стеклах оптического кварца растворимость гелия почти на четыре порядка выше и составляет $(7.01 \pm 0.04) \cdot 10^{19} \mathrm{~cm}^{-3}$ при $P=25.5 \mathrm{MPa}$ и $T=373 \mathrm{~K}$.

Вопрос влияния включений в монокристаллах кварца на растворимость гелия требует дополнительных исследований.

\section{Список литературы}

[1] Mamyrin B.A., Anufriev G.S., Kamensky U.L. et al. // Geochem. Int. 1970. Vol. 7. P. 498.

[2] Жиганов А.Н., Купряжкин А.Я., Мулюков Р.Р. и др. // ЖТФ. 2002. Т. 72. Вып. 11. С. 96-99.

[3] Barrer R.M., Vaughan E.W. // Trans. Faraday. Soc. 1967. Vol. 63. P. 2275.

[4] Веттегрень В.И., Соболев Г.А., Киреенкова С.М. и др. // ФТТ. 2014. Т. 56. Вып. 6. С. 1180.
[5] Аргунова Т.С., Сорокин Л.М., Певзнер Б.З. и др. // ФТТ. 2003. Т. 45. Вып. 10. С. 1818.

[6] Swets D.E., Lee R.W., Frank R.C. // J. Chem. Phys. 1961. Vol. 34. N 1. P. 17.

[7] Купряжкин А.Я., Поликарпов Ф.Д., Попов Е.В. // ЖФХ. 1981. T. 55. № 5. C. 1238.

[8] Jones W.M. // J. Am. Chem. Ser. 1953. Vol. 75. P. 3093.

[9] Купряжкин А.Я., Куркин А.Ю. // ФТТ. 1993. Т. 35. Вып. 11. C. 3003. 